\title{
Zodiacal Light Surface Brightness Measurements by Atmosphere Explorer-C
}

\author{
MARSHA R. TORR*, D. G. TORR*, AND R. STENCEL $\dagger$ \\ Space Physics Research Laboratory, University of Michigan, Ann Arbor, Michigan 48109
}

Received October 13, 1977; revised February 13, 1979

\begin{abstract}
Using the visible airglow photometer on the Atmosphere Explorer-C satellite, we have mapped the zodiacal light surface brightness at the wavelengths monitored by the instrument: 3371,4278 , $5200,5577,6300$, and $7319 \AA$. The study constitutes a survey over this wavelength range, covering most of the celestial sphere, from altitudes above the atmospheric emissions, and free from atmospheric scattering and attenuation. The intensity variations reveal enhancements near elongations of $130^{\circ}$, and possibly near $60^{\circ}$, at all wavelengths. The intensity of the zodiacal light near the ecliptic pole is found to be $\sim 30 S_{10}$. The color ratio with respect to the Sun is found to be redder than the Sun (0.7) at all elongations.
\end{abstract}

\section{INTRODUCTION}

Much of our knowledge of dust and particulate matter in the solar system comes from observations of sunlight scattered off these particles. An addition to the data base of surface brightness measurements that have been accumulated over the years is the large volume of measurements, made at high altitude and at several wavelengths, by the Visible Airglow Experiment (VAE) on the Atmosphere Explorer satellites (Hays et al., 1973).

Satellite measurements of the zodiacal light have an advantage over ground-based measurements because of freedom from contamination by atmospheric emissions and the problems of scattering and extinction in the lower atmosphere.

The AE satellites have two modes of operation: despun, and spinning about an axis normal to the orbital plane. This motion, together with the precession of the orbital plane, allows almost complete coverage in ecliptic latitude and longitude. In this paper we present maps of the surface brightness

\footnotetext{
* Also affiliated with the National Institute for Telecommunications Research of the C.S.I.R., Johannesburg, South Africa.

† Present address: Goddard Space Flight Center, Greenbelt, Md. 20771.
}

as a function of ecliptic latitude $(\beta)$ and heliocentric ecliptic longitude ( $\epsilon$ ) for 3371 , 4278, 5200, 5577, 6300, and $7319 \AA$. The results are compared with a Mie-type scattering model and with previous measurements. We determine color ratios, relative to the Sun, for several pairs of wavelengths.

\section{MEASUREMENTS}

The surface-brightness measurements used in this paper were made by the visible airglow photometer on the AE-C satellite. This instrument has been described by Hays et al. (1973). The photometer contains six interference filters, with bandwidths of $\sim 20 \AA$, mounted in a filter wheel so that any one of these can be moved into the optical path. In addition, background and calibration positions are available. The photometer has two optical channels, oriented at right angles to the spin axis. The measurements discussed here were made with the wider of these channels, which had a half-angle field of view of $3^{\circ}$.

The data base analyzed in what follows was accumulated over a 10 -month period between January and October 1974, during which the AE-C was in an elliptic orbit, and measurements were made from altitudes above the atmospheric emissions. In a separate paper (Torr et al., 1977) we have 


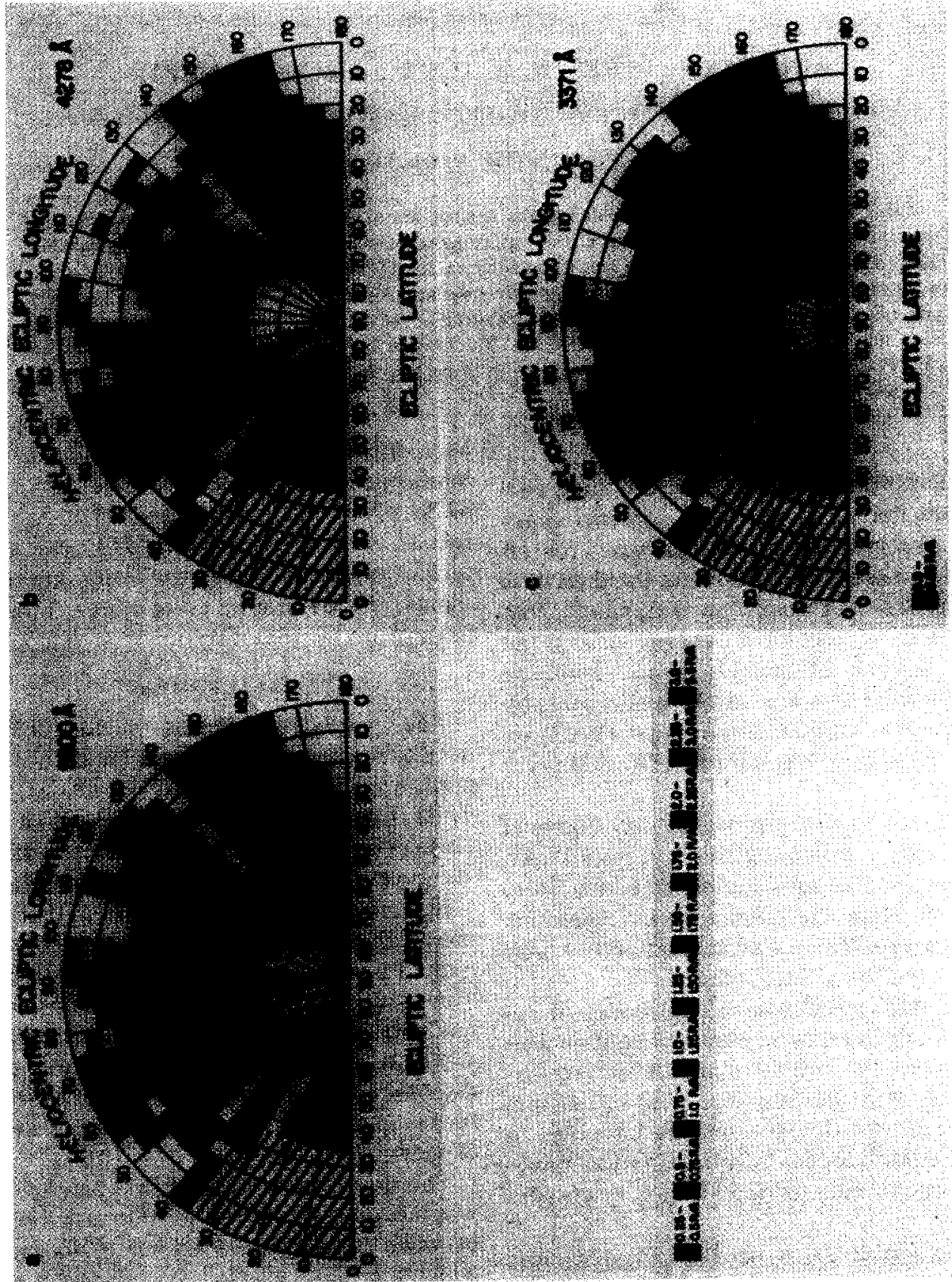



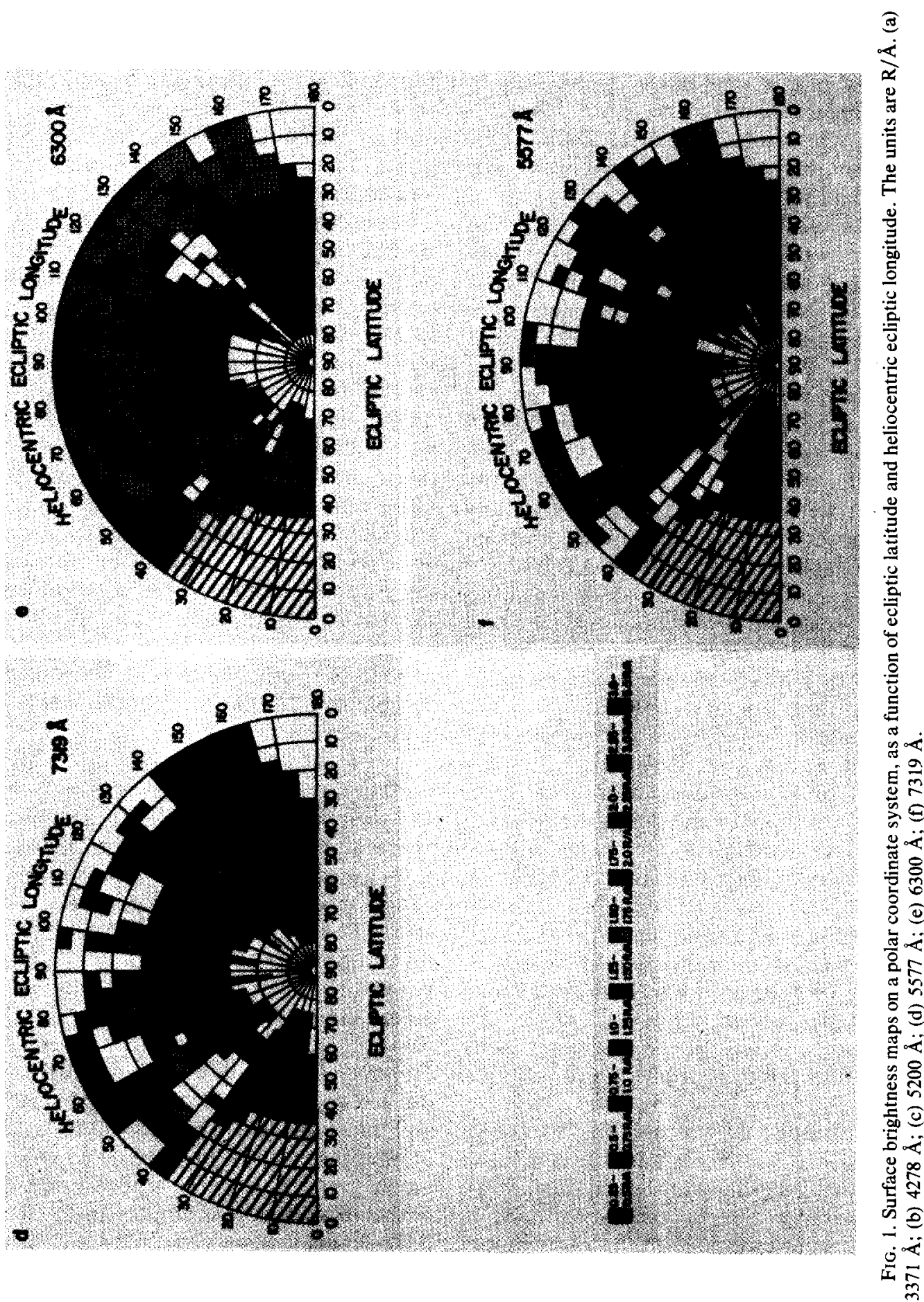
addressed the question of stability of the sensitivity calibration of the instrument over this time period. By monitoring an inflight calibration source (as a check on the stability of the photometer gain), and by performing some coordinated measurements with ground-based stations, we are confident that the sensitivity did not change significantly.

Considerable care has been taken to avoid measurements made under any conditions that might introduce spurious data. Some of these selection constraints have been discussed in a separate paper (Torr et al., 1977). We have not used measurements made below $500 \mathrm{~km}$ in altitude on the day side, and below $450 \mathrm{~km}$ on the nightside. In addition, we have used only data measured when the photometer was looking into the upward hemisphere, so as to avoid any slant observing paths downward through the atmosphere. The AE photometers have a two-stage baffle system, designed to attenuate scattered light from outside the field of view by $10^{12}$ (Hays et al., 1963; Torr et al., 1977). This system makes it possible to measure the zodiacal light at a surface brightness of $\sim 1 \mathrm{R} / \AA$ in the presence of sunlight $\left(\sim 5 \times 10^{11} \mathrm{R} / \AA\right.$ if viewed $\mathrm{di}$ rectly). We have not used data taken closer than $35^{\circ}$ to the Sun and $20^{\circ}$ to the Moon.

We have avoided measurements made in the vicinity of the South Atlantic Radiation Anomaly by rejecting any data between geographic longitudes $100^{\circ} \mathrm{W}$ to $50^{\circ} \mathrm{E}$, and between geographic latitudes $15^{\circ} \mathrm{N}$ to $55^{\circ} \mathrm{S}$. Outside this region, we have not used data at altitudes above $1000 \mathrm{~km}$, where fast charged particles in the radiation belts might affect the instrument (Torr et al., 1977).

The attitude of the satellite is known nominally to $\pm 1^{\circ}$. From this information we have calculated the viewing direction of the photometer in ecliptic coordinates and in galactic coordinates. For the purposes of the zodiacal maps, we have not used data within $30^{\circ}$ of the galactic equator. (The galactic component has also been mapped and will be reported separately.)

Thus sorted, the data have been averaged into $5^{\circ} \times 5^{\circ}$ bins. The results are shown in Fig. 1 (a-f). We have assumed symmetry about the Sun in the ecliptic plane, and have averaged northern and southern ecliptic latitudes, and east and west heliocentric longitudes. This is duscussed further below.

Some gaps exist in the coverage. As a routine operational precaution, the photometer was not turned on when the orbital plane was within $\sim 12^{\circ}$ of the Sun. A result of this was that no data were obtained within $12^{\circ}$ of the antisolar direction, precluding mapping of the gegenschein. The orbital inclination of the AE-C satellite was $68.4^{\circ}$. This precluded observations being taken in the vicinity of the ecliptic pole. Other unshaded areas of the maps were left unfilled either because the sample was small $(<10)$ or the standard deviation was large $(>40 \%)$. Each $5^{\circ}$ bin is therefore an average of anywhere between 10 and several hundred individual measurements. We hope to complete the maps at a later date by combining information from the AE-D and AE-E satellites.

As the data have been carefully screened to avoid atmospheric emissions and radiation belt effects, the residual major nonzodiacal components are due to individual stars and diffuse starlight. We consider the effects of each of these in turn. For an instrument operated from a platform that varies slowly with time, such as a groundbased instrument, a first-magnitude star in a $3^{\circ}$ half-cone angle field of view would contribute $\sim 134 \quad S_{10}(V)$. A contribution of this magnitude would be comparable with the zodiacal component at large elongations and thus would require removal for studies of these regions. However, the effect is much less severe for a spinning satelliteborne instrument that progressively sweeps the celestial sphere. First, the spinning motion of the vehicle effectively smears the field of view over the integration period, so 
that it is $\sim 54$ square degrees, rather than 28 (for a $3^{\circ}$ half-cone angle). The contribution of a first-magnitude star is thus $\leq 70 \mathrm{~S}_{10}(V)$ rather than $134 \mathrm{~S}_{10}(V)$. A more significant point, however, is the fact that the star is only in the field of view for $\leqslant 0.13 \mathrm{sec}$, the duration of the integration period. The next time (days, weeks, or months later) that the same $5^{\circ} \times 5^{\circ}$ portion of the zodiacal light is scanned by the instrument, the stellar background will be different. On the average $\sim 100$ integration periods (accumulated over the 10 months of measurement) comprise an average surface brightness shown in a given $5^{\circ} \times 5^{\circ}$ bin at a particular wavelength. Thus the contribution of the first-magnitude star is reduced to $\sim 0.7 \mathrm{~S}_{10}(V)$ which corresponds to $3 \times 10^{-3} \mathrm{R} / \AA$ at $5577 \AA$ and is not significant. Stars of smaller magnitude will not be detectable. Only large magnitude stars that were encountered on successive spins would degrade the data, and the number of such stars and the nature of the observations makes this source of error small for the purposes of this study.

We consider next the contribution from diffuse starlight. According to Shectman (1974) and Roach and Megill (1961), the contribution from numerous faint-field stars at high galactic altitudes is $\sim 28 \mathrm{~S}_{\mathbf{1 0}}(V)$. Diffuse galactic light above $b=30^{\circ}$ amounts to $\sim 20 \mathrm{~S}_{10}(V)$ (Roach and Smith, 1964). Thus the total is $\sim 48 \mathrm{~S}_{10}(V)$ which is less than $0.2 \mathrm{R} / \AA$ at $5577 \AA$. This contribution is $25 \%$ of the lowest surface brightness level shown in the zodiacal maps at elongations. At smaller elongations, the contribution is not significant.

\section{COMPARISON OF RESULTS WITH EARLIER WORK}

The most recent review on the zodiacal light is that by Leinert (1975), and we refer readers to this work for a bibliography of papers in the field. In this section we shall compare the data shown in Fig. 1 with results previously published, and address some of the main issues.

\subsection{Variation in the Ecliptic Plane}

The surface brightnesses shown in Fig. 1 are in units of rayleighs per angstrom, $R / \AA$ (a unit commonly used in aeronomy, where $1 \mathrm{R}=10^{6} / 4 \pi$ photons $\mathrm{cm}^{-2} \mathrm{sec}^{-1} \mathrm{sr}^{-1}$ ). The most commonly used unit in zodiacal light work is $S_{10}$, the number of tenth-magnitude stars per square degree. In Table I we show a conversion between these two units at the wavelengths considered in this paper.

Much of the earlier work has been restricted to the plane of the ecliptic. Leinert (1975) has shown a comparison of intensity measurements made by 16 workers since 1964 , as a function of elongation, $\epsilon^{\prime}$ (where $\epsilon^{\prime}$ is equivalent to heliocentric longitude, $\epsilon$, in the ecliptic plane; $\cos \epsilon^{\prime}=\cos \epsilon \cos \beta$ ). In Fig. 2 we show the variation of the $\mathrm{AE}$ data for the six wavelengths analyzed here, and we have superimposed on this the envelope of the spread of the earlier data.

The AE data are in general agreement with the earlier observations. However, in Fig. 2 the AE observations show an enhancement near $\epsilon \sim 130^{\circ}$. This can also be seen in Fig. 1, and extends to higher latitudes. A comparable feature was indicated in the data of Smith, et al. (1975) and also in the data presented by Chiplonkar and Tillu (1967), but is generally absent from the results shown by other workers. We discuss this further in Section 3.3.

As we mentioned earlier the data maps shown in Fig. 1 assume symmetry about the Sun in the ecliptic plane. Several analyses have concluded that the axis of symmetry is

TABLE I

\begin{tabular}{cc}
\hline$\lambda$ & $\begin{array}{c}\text { No. of } S_{10} \\
\text { per 1 R/ } \AA\end{array}$ \\
\hline 3371 & 772 \\
4278 & 334 \\
5200 & 242 \\
5577 & 234 \\
6300 & 229 \\
7319 & 244 \\
\hline
\end{tabular}




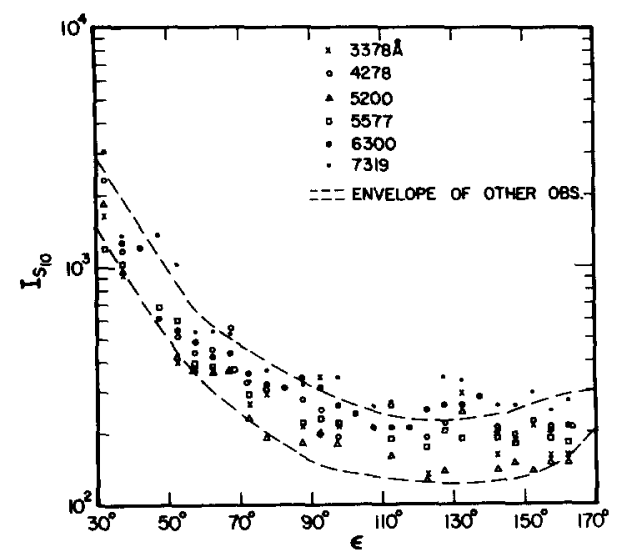

FIG. 2. Variation of zodiacal light surface brightness vs heliocentric ecliptic longitude for the six wavelengths analyzed. Also shown (dotted lines) is the envelope of a compilation of measurements made since 1964. For the sources of these see Leinert (1975).

the invariable plane rather than the ecliptic plane. The inclination, however, is smaller than the bin size selected for averaging the data shown here. An inspection of the data from the northern and southern hemispheres does not reveal any hemispherical asymmetry detectable within the statistical variation of the data. This is in agreement with measurements by Sparrow and Ney (1972) and Leinert et al. (1974). An eastwest symmetry is generally assumed to exist, corresponding to the rotational symmetry of the cloud. In this respect, these data are in agreement with findings by Frey et al. (1974), Dumont (1965), and Leinert et al. (1974) which confirm this symmetry. We therefore do not believe that the averaging has introduced errors of significant magnitude for the purpose of this study.

\subsection{Brightness Near the Ecliptic Pole}

Earlier measurements of the surface brightness of the zodiacal light near the ecliptic pole have ranged from 50 to $123 \mathrm{~S}_{10}$. As was mentioned earlier, the AE-C photometer was not able to make measurements right at the ecliptic pole. However, we did obtain data $\sim 10^{\circ}$ away, and the scale of the variations is such that we can obtain from this an estimate of the brightness at the pole. In the case of $6300 \AA$, we estimate $60 S_{10}$ uncorrected for star background. From the tables by Roach (1960) and Roach and Megill (1961), the integrated starlight in this region should be $\sim 30 \mathrm{~S}_{10}$. We therefore conclude from the $\mathrm{AE}$ data that the zodiacal component in the vicinity of the ecliptic pole is $\sim 30 \mathrm{~S}_{10}$. The data are sparse at high latitudes. However, no significant northsouth asymmetry is apparent at $20-30^{\circ}$ from the poles.

\subsection{Intensity Variations out of the Ecliptic}

In Section 3.1 we compared the AE surface brightness measurements in the ecliptic plane with earlier measurements. We pointed out an enhancement near $\epsilon \sim 130^{\circ}$ and mentioned that this feature (present at all the wavelengths studied) extended to higher ecliptic latitudes. The feature appears to be related to elongation. A second, although more subtle, enhancement can be seen in Fig. 1, at higher latitudes near $\epsilon \sim 60^{\circ}$. This appears to be the feature reported by Smith et al., (1965), but which has been largely ignored because it was not evident in most other observations. In Fig. 3 we compare our results at $6300 \AA$ with the isophotes shown by Frey et al. (1974). The feature near $\epsilon^{\prime} \sim 60^{\circ}$ would not have easily emerged from the Frey et al. results because of the low $\epsilon^{\prime}$ cutoff in their data. However, the enhancement near $\epsilon^{\prime} \sim 130^{\circ}$ in the AE data, corresponds to a very definite minimum in the Frey et al. data. (It should be noted for the purposes of this comparison, that the AE data are not corrected for diffuse starlight.) Also, the enhancement seen in the $\mathrm{AE}$ data near $\epsilon^{\prime} \sim 60^{\circ}$, only emerges at higher ecliptic latitudes and at longitudes for which Frey et al. do not show data.

Clearly, if real, these features contain very significant information about the nature of the interplanetary dust. We have therefore devoted considerable effort in attempting to ascertain whether the enhancements are real or artifacts of the data. As was mentioned earlier, we have not in- 


\section{HELIOCENTRIC ECLIPTIC LONGITUDE}

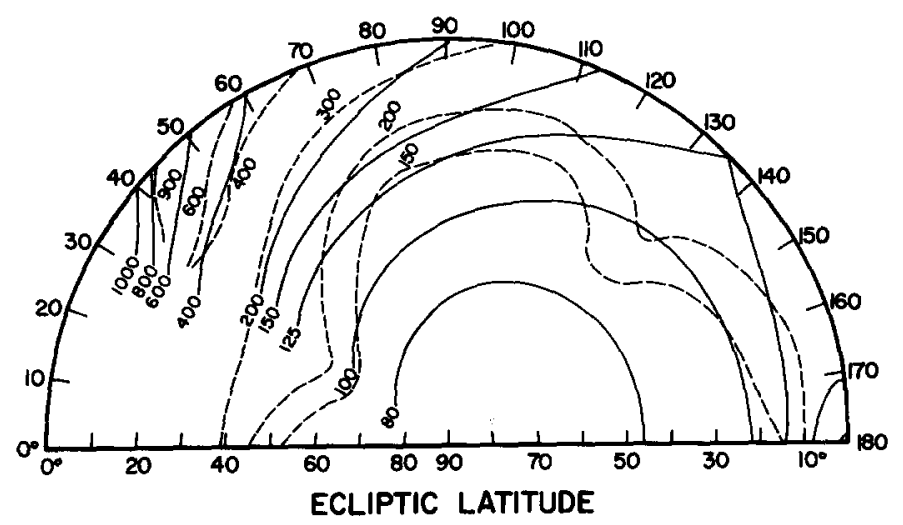

FIG. 3. Isophotes (in $S_{10}$ ) of the AE measurements at $6300 \AA$ superimposed on the measurements made by Frey et al., (1974).

cluded in the surface brightness data shown here, any averages for which the standard deviation was $\geq 40 \%$ or the number of samples $<10$. From an examination of the data in the enhancement regions, it does not appear that these brighter regions are due to undersampling.

A possible source of these brighter regions might be stars in the field of view of the instrument. We have discussed this question earlier and generally bright stars would not degrade the observations unless present in the field of view for a significant number of the samples comprising the average brightness in any given bin. The possibility of this occurring is small, but in case this might contribute to the enhancements in question, we have analyzed all the data falling in the region of the enhancements on an orbit-by-orbit basis. We have mapped the viewing direction in right ascension and declination. In each case where a star could possibly have been in the field of view, we have determined the difference between the surface brightness in the enhancement region and that of the surrounding regions. We have plotted this difference vs the apparent magnitude of the star (Fig. 4). The results indicate that only the very brightest stars $(V<1.0 \mathrm{~m})$ obey the usual relation between flux and apparent magnitude $m \propto 2.5 \log F$.

Stars fainter than $V=1.0$ do not appear to dominate the extended surface brightness in the field of view. We therefore conclude that the enhancements are due to real features of the zodiacal light, but we require further measurements in order to confirm this.

We shall comment further on these regions in the following section.

\subsection{Comparison with Theory}

Models of the zodiacal light surface

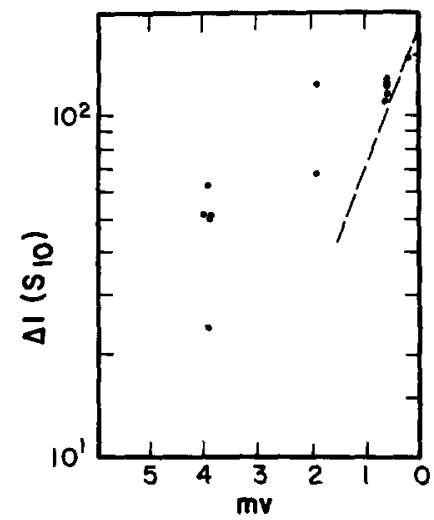

FIG. 4. The points show the surface-brightness enhancements at times when stars were in the field of view. The dashed line shows the response anticipated from the $m \alpha 2.5 \log F$ relationship, if the stellar contribution dominated the signal. 
brightness are generally based on Mie scattering theory (scattering by spherical particles). Such models are clearly approximations, in that the particles are not perfect spheres. However, they do allow useful first-order modeling of the large-scale variations of the dust cloud. Because so many models have appeared (and are appearing) in the literature based on this approach, in this section we compare the AE data with Mie theory. Such models assume a power law for the dependence of particle number density, $N$, on solar distance, $r$,

$$
d N \simeq r^{-\nu} \alpha^{-k} d \alpha,
$$

where $\nu$ is the exponent of the radial density distribution, $\alpha$ is the size parameter

$$
\alpha=2 \pi a / \lambda,
$$

and $a$ is the radius of the particles.

Using the notation of Aller et al. (1967), illustrated in Fig. 5, the surface brightness of the zodiacal light at a particular wavelength is given by

$$
\begin{array}{r}
I_{\lambda}{ }^{i} d \omega=E_{\lambda}\left(\lambda^{2} / 8 \pi^{2}\right) \int_{0}^{\infty}\left(R_{0}{ }^{2} / \rho^{2}\right) \\
N(r, z) \sigma_{i}(\Theta, k) d \Delta d \omega .
\end{array}
$$

$I_{\lambda}{ }^{i}$ is the monochromatic solar flux at the Earth. Introducing an average density distribution given by

$$
N(r, z)=N_{0}\left(R_{0} / r\right)^{v} \exp \left(-K z / R_{0}\right),
$$

where $N_{0}$ is the density of scatterers at $R_{0}$, and performing several angular transformations, Aller et al. (1967) show that

$$
\begin{aligned}
& I_{\lambda}^{i}(\epsilon, \beta)=E_{\lambda} N_{0} R_{0}\left(\lambda^{2} / 8 \pi^{2}\right) \\
& \int_{\epsilon}^{\pi}\left[(\sin \theta)^{\nu} / \epsilon^{\prime}\left(\sin \epsilon^{\prime}\right)^{\nu}+(\cos l)^{\nu}\right] \\
& \cdot \sigma_{i}(\theta, m, k) \times \exp [-K\{[\sin (\theta \\
& \left.\left.\left.\left.-\epsilon^{\prime}\right)\right] / \sin \theta\right\} \sin \beta\right] d \theta,
\end{aligned}
$$

where $K$ is the scale height factor (in AU) in the density distribution above the ecliptic plane, $\sigma(\Theta, m, k)$ is the scattering cross section, and $m(\lambda)$ is the complex index of refraction of the material.

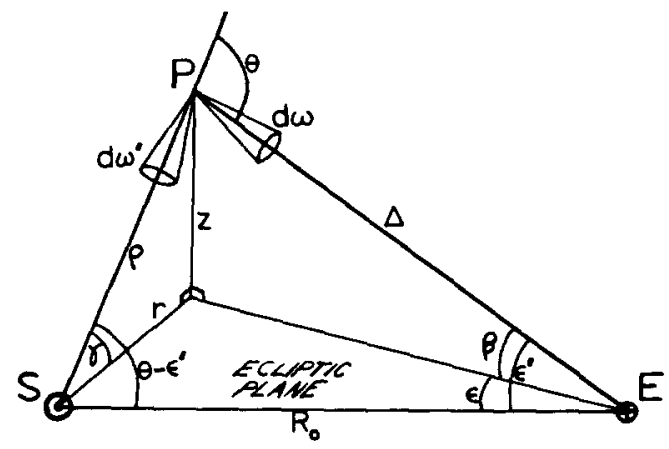

Fig. 5. From Frey et al. (1967). Relation between Sun-particle distance, $\rho$, particle-Earth distance $\Delta$, and scattering angle $\theta$. S represents Sun, E, Earth, and $P$, scattering particle.

Giese (1972) has published the results of models for a range of sizes with $\alpha_{\min } \leq \alpha \leq 120 \quad\left(\alpha_{\min }=1,2,4,10,60\right)$, for flat $(k=2.5)$ and steep $(k=4)$ size spectra, and complex refractive indices $m=$ $m_{1}-m_{2} i$ with $m_{1}=1.33,1.5,1.7$ and $m_{2}=0,0.01,0.05$, and 0.1. A scan of his numerous plots of the calculated surface brightness vs $\epsilon$ shows that he is able to reproduce the enhancement at $\epsilon \simeq 130^{\circ}$ using $m=1.33, \alpha=60-120$, for $k=2.5$ or 4.0 .

We have similarly modeled the zodiacal surface brightness as a function of $\epsilon^{\prime}$ and $\beta$, and in Fig. 6 we show illustrative results in the same polar coordinate system as we have shown the data. In these calculations we have used a radial density distribution in accordance with the Pioneer measurements of Weinberg (1976), namely, $\mathrm{r}^{-1}$. The scale height factor is rather less well determined. Aller et al. (1967) use $K=5$, while Dumont (1975) derives an ellipsoidal cloud model of axial ratio $7: 1$. Our calculations indicate that $K$ between 5 and 6 adequately matches the $I_{\lambda}(\epsilon, 0) / \epsilon$ slope for $35^{\circ}<\epsilon<90^{\circ}$. We have used $\sigma(\Theta)$ presented by Geise (1972) for size distribution defined by $d N \propto \alpha^{-k} d \alpha$ for $60 \leq \alpha \leq 120 \mu, k=4.0$, for Mie theory calculations with index of refraction $m=1.33$ and $m=1.33-0.01 i$.

The enhancement near $\epsilon^{\prime} \sim 130^{\circ}$ can be seen in these results, and if real indicates larger particles with $m \simeq 1.33$. The $\mathrm{AE}$ 


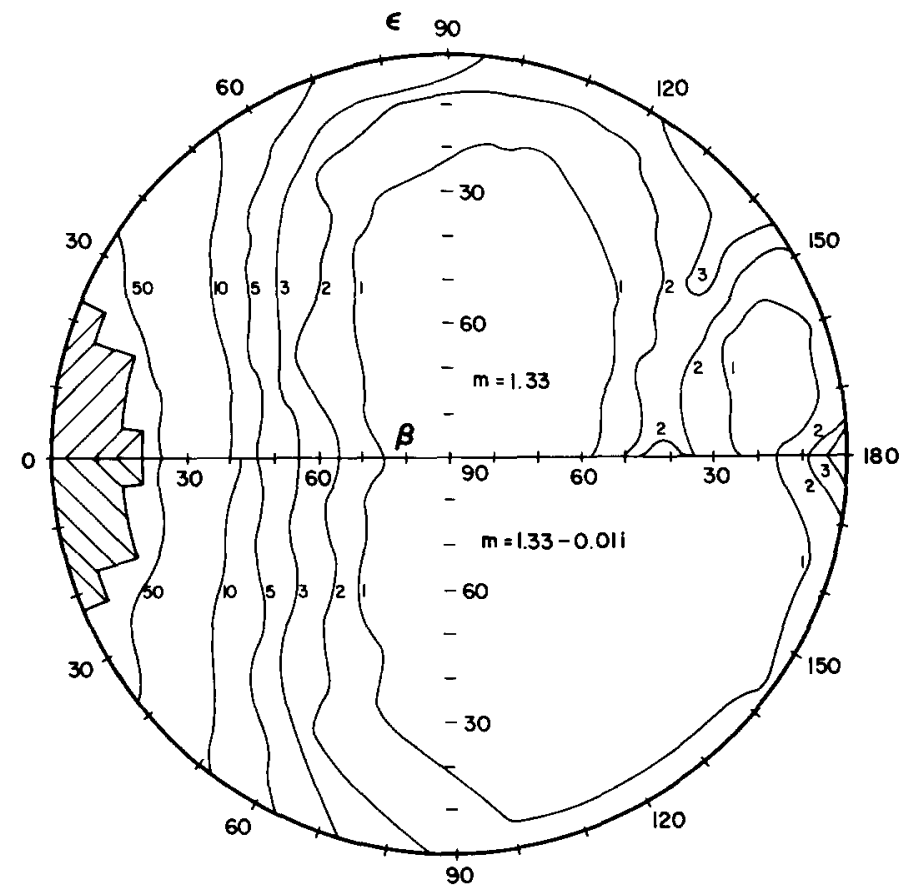

FIG. 6. Isophotes of surface brightness calculated from Mie-type scattering theory. The upper half is for $m=1.33$ and the lower half for $m=1.33-0.01 i$ (relative units).

data, however, also indicate a second bright feature near $\epsilon^{\prime} \sim 60^{\circ}$ and this does not emerge from the theoretical calculations. These features would correspond to scattering angles of $150-160^{\circ}$. We may therefore be seeing a halo effect associated with the peaks in the scattering cross section near these angles.

\subsection{Color of Zodiacal Light}

The spectral distribution of the zodiacal light is typically compared with that of the Sun. This is because departures from the solar color would directly reflect the size distributions of the particulate matter. Previous reports have ranged from redder than the Sun to bluer than the Sun, and some have found the color ratio to vary with elongation (Leinert et al., 1974). We can define the color ratio relative to the Sun by

$$
\begin{aligned}
& C\left(\lambda_{1}, \lambda_{2}\right)= \\
& {\left[I_{Z L}\left(\lambda_{1}\right) / I_{\odot}\left(\lambda_{1}\right)\right] /\left[I_{Z L}\left(\lambda_{2}\right) / I_{\odot}\left(\lambda_{2}\right)\right]}
\end{aligned}
$$

and using this, we can determine the color ratio from the data contained in Fig. 1 for various elongations. In Fig. 7 we show the results for eight wavelength pairs. As can be seen from the figure, the color ratio with respect to the Sun is found to be 0.7 $( \pm 20 \%)$, i.e., redder than the Sun, for eight color pairs, and for all elongations. Leinert et al. (1974) found a reddening of the zodiacal light, smaller than that shown here, for $e<40^{\circ}$. Also, indications of a reddening of the zodiacal light have been reported in the corona [see, for example, Gillett et al. (1964)]. However, Frey et al., (1974) found no deviation from solar color for $\epsilon>45^{\circ}$. As has been discussed earlier, the AE results have a residual component due to starlight amounting to $\sim 30 \%$ for $\epsilon \gtrsim 90 \%$. Thus at larger elongations, the reddening found in Fig. 7 may be enhanced by the stellar component. However, for smaller elongations, the stellar component should not be significant. 


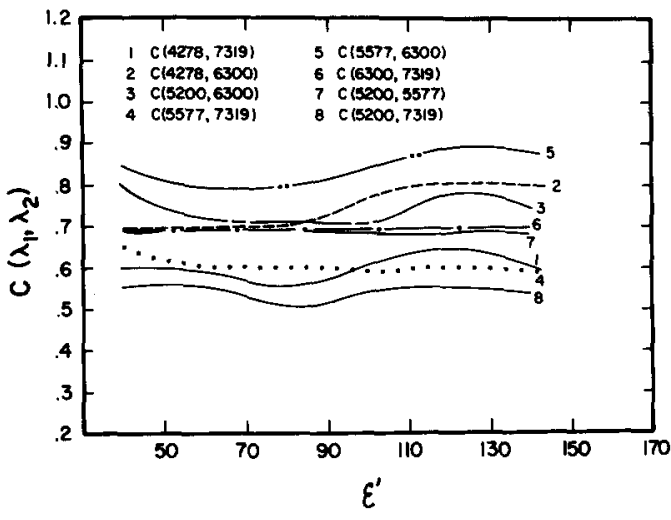

FIg. 7. Average color ratio with respect to the Sun for eight wavelength pairs, as a function of elongation.

\section{CONCLUSIONS}

We have presented here maps of the zodiacal light surface brightness as a function of ecliptic latitude and heliocentric ecliptic longitude, at six wavelengths ranging from the near ultraviolet to the near infrared. The measurements are made from above the atmosphere from the Atmosphere Explorer-C satellite. This study constitutes the first phase of the reduction of these data and some initial findings are presented. A second phase will attempt to achieve a better separation of the zodiacal and stellar components. However, for the purposes of this study, the stellar contribution is less than $30 \%$ of the intensities shown in the maps.

The AE data have produced intensity variations that are basically in accord with earlier measurements in the plane of the ecliptic. However, fairly subtle enhancements are found in two regions of the celestial sphere at all the wavelengths analyzed. Attempts to explain these enhancements in terms of artifacts in the data or contamination by other sources have not been successful. We are therefore left with the conclusion that the enhancements may be real features of the zodiacal light. The enhancement near $\epsilon \simeq 130^{\circ}$ is the more distinctive of the two. This can be reproduced in a Mie-type scattering model by using $m=1.33,60 \leq \alpha \leq 120$, and $k=2.5$ or 4.0, but other explanations may exist.

The intensity near the ecliptic pole agrees with the lower range of values reported by other workers, i.e., $\sim 30 \mathrm{~S}_{10}$. The color ratio with respect to the Sun is found to be $\sim 0.7$, i.e., redder than the Sun, for eight color pairs and for all elongations.

\section{ACKNOWLEDGMENTS}

We are grateful to Dr. P. B. Hays for the Atmosphere Explorer-C photometer data, from which this study was made. This research was supported at the University of Michigan by Grant NAS5-24331.

\section{REFERENCES}

Aller, L. H., Duffner, G., Dworetsky, M., Gudehus, D., Kilston, S., Leckrone, D., Montgomery, J., Oliver, J., ANd Zimmerman, E. (1967). In Zodiacal Light and Interplanetary Medium (J. L. Weinberg, Ed.), p. 243. NASA SP-150.

Chiplonkar, M., And Tillu, A. (1967). Photometric evaluation of the zodiacal light and gegenschein. Ann. Geophys. 23, 17.

Dumont, R. (1975). Ground-based observations of the zodiacal light. In Interplanetary Dust and Zodiacal Light (H. Elsasser and H. Fechtig, Eds.), p. 85. IAU Colloquium 31, Springer-Verlag, Berlin.

Frey, A., Hofmann, W., Lemke, D., and Thum, C. (1974). Photometry of the zodiacal light with balloon-borne telescope THISBE. Astron. Astrophys. 36, 447.

GiEsE, R. (1972). Single component zodiacal light models. Dudley Obs. Report No. 7.

Gillett, F. C., Stein, W. A., And Ney, E. P. (1964). Observations of the solar corona from the limb of the sun to the zodiacal light, July 20,1963. Astrophys. $J$. 140, 292.

Hays, P. B., Carignan, G. R., Kennedy, B. C., Shepherd, G. G., ANd WAlker, J. C. G. (1973). The visible-airglow experiment on atmosphere explorer. Radio Sci. 8, 369-378.

LeINERT, C. (1975). Zodiacal light. Space Sci. Rev. 18, 281-339.

Leinert, C., Link, H., and Pitz, E. (1974). The thermal emission of the dust corona during the eclipse of June 30, 1973. Astron. Astrophys. 37, 81.

RoACH, F. E. (1960). Diffuse galactic light. In Modern Astrophysics. Gauthier-Villars, Paris.

RoACH, F. E., AND Megill, L. R. (1961). Integrated starlight over the sky. Astrophys. J. 133, 228.

Shectman, S. A. (1974). The small scale anisotropy of the cosmic light. Astrophys. J. 188, 233.

SMith, L. L., RoAch, F. E., AND OWEN R. W. (1965). Absolute photometry of the zodiacal light. Planet. Space Sci. 13, 207-217. 
Sparrow, J. G., AND Ney, E. P. (1972). Observations of the zodiacal light from the ecliptic to the poles. Astrophys, J. 174, 705.

TorR, M. R., HaYs, P. B., Kennedy, B. C., AND WALKER, J. C. G. (1977). Intercalibration of airglow observations with the Atmosphere Explorer satellite. Planet. Space Sci. 25, 173-184.

WEINBERG, J. L. (1976). Light of the night sky. In $I A U$ Reports of Astronomy, (G. Contopoulos, Ed.), Vol. 1, p. 135. Reidel, Dordrecht. 